\title{
Male breast cancer in BRCA1 and BRCA2 mutation carriers: pathology data from the Consortium of Investigators of Modifiers of BRCA1/2
}

Valentina Silvestri1 ${ }^{1 \dagger}$, Daniel Barrowdale ${ }^{2 \dagger}$, Anna Marie Mulligan, ${ }^{3,4}$, Susan L. Neuhausen ${ }^{5}$, Stephen Fox ${ }^{6}$, Beth Y. Karlan, Gillian Mitchell ${ }^{8,9}$, Paul James ${ }^{8,9}$, Darcy L. Thull ${ }^{10}$, Kristin K. Zorn ${ }^{10}$, Natalie J. Carter ${ }^{11}$, Katherine L. Nathanson ${ }^{12}$, Susan M. Domchek ${ }^{12}$, Timothy R. Rebbeck ${ }^{13}$, Susan J. Ramus ${ }^{14}$, Robert L. Nussbaum ${ }^{15}$, Olufunmilayo I. Olopade ${ }^{16}$, Johanna Rantala ${ }^{17}$, Sook-Yee Yoon ${ }^{18,19}$, Maria A. Caligo ${ }^{20}$, Laura Spugnesii ${ }^{20}$, Anders Bojesen ${ }^{21}$, Inge Sokilde Pedersen ${ }^{22}$, Mads Thomassen ${ }^{23}$, Uffe Birk Jensen ${ }^{24}$, Amanda Ewart Toland ${ }^{25}$, Leigha Senter ${ }^{26}$, Irene L. Andrulis, ${ }^{4,27}$, Gord Glendon ${ }^{27}$, Peter J. Hulick ${ }^{29}$, Evgeny N. Imyanitov ${ }^{30}$, Mark H. Greene ${ }^{31}$, Phuong L. Mai ${ }^{31}$, Christian F. Singer ${ }^{32}$, Christine Rappaport-Fuerhauser ${ }^{32}$, Gero Kramer ${ }^{33}$, Joseph Vijai ${ }^{34}$, Kenneth Offit ${ }^{34}$, Mark Robson ${ }^{35}$, Anne Lincoln ${ }^{34}$, Lauren Jacobs ${ }^{34}$, Eva Machackova ${ }^{36}$, Lenka Foretova ${ }^{37}$, Marie Navratilova ${ }^{36}$, Petra Vasickova ${ }^{36}$, Fergus J. Couch ${ }^{38,39}$, Emily Hallberg ${ }^{39}$, Kathryn J. Ruddy ${ }^{40}$, Priyanka Sharma ${ }^{41}$, Sung-Won Kim ${ }^{42}$, kConFab Investigators, Manuel R. Teixeira ${ }^{43,44}$, Pedro Pinto ${ }^{43}$, Marco Montagna ${ }^{45}$, Laura Matricardi ${ }^{45}$, Adalgeir Arason ${ }^{46}$,

Oskar Th Johannsson ${ }^{47}$, Rosa B. Barkardottir ${ }^{46}$, Anna Jakubowska ${ }^{48}$, Jan Lubinski ${ }^{48}$, Angel Izquierdo ${ }^{49}$, Miguel Angel Pujana ${ }^{50}$, Judith Balmaña ${ }^{51}$, Orland Diez ${ }^{52}$, Gabriella Ivady ${ }^{53}$, Janos Papp ${ }^{54}$, Edith Olah ${ }^{54}$, Ava Kwong ${ }^{55,56}$, Hereditary Breast and Ovarian Cancer Research Group Netherlands (HEBON), Heli Nevanlinna ${ }^{57}$, Kristiina Aittomäki ${ }^{58}$, Pedro Perez Segura ${ }^{59}$, Trinidad Caldes ${ }^{60}$, Tom Van Maerken ${ }^{61}$, Bruce Poppe ${ }^{61}$, Kathleen B. M. Claes ${ }^{61}$, Claudine Isaacs ${ }^{62}$, Camille Elan ${ }^{63}$, Christine Lasset ${ }^{64,65}$, Dominique Stoppa-Lyonnet ${ }^{63,66}$, Laure Barjhoux ${ }^{67}$, Muriel Belotti ${ }^{63}$, Alfons Meind ${ }^{68}$, Andrea Gehrig ${ }^{69}$, Christian Sutter ${ }^{70}$, Christoph Engel ${ }^{71}$, Dieter Niederacher ${ }^{72}$, Doris Steinemann ${ }^{73}$, Eric Hahnen ${ }^{74}$, Karin Kast ${ }^{75}$, Norbert Arnold ${ }^{76}$, Raymonda Varon-Mateeva ${ }^{77}$, Dorothea Wand ${ }^{78}$, Andrew K. Godwin ${ }^{79}$, D. Gareth Evans ${ }^{80}$, Debra Frost ${ }^{2}$, Jo Perkins², Julian Adlard ${ }^{81}$, Louise Izatt ${ }^{82}$, Radka Platte ${ }^{83}$, Ros Eeles ${ }^{84}$, Steve Ellis ${ }^{2}$, EMBRACE, Ute Hamann ${ }^{84}$, Judy Garber ${ }^{85}$, Florentia Fostira ${ }^{86}$, George Fountzilas ${ }^{87}$, Barbara Pasini ${ }^{88,89}$, Giuseppe Giannini ${ }^{1}$, Piera Rizzolo ${ }^{1}$, Antonio Russo ${ }^{90}$, Laura Cortesi ${ }^{91}$, Laura Papi ${ }^{92}$, Liliana Varesco ${ }^{93}$, Domenico Pallii ${ }^{94}$, Ines Zanna ${ }^{94}$, Antonella Savarese ${ }^{95}$, Paolo Radice ${ }^{96}$, Siranoush Manoukiann ${ }^{97}$, Bernard Peissel ${ }^{97}$, Monica Barile ${ }^{98}$, Bernardo Bonanni ${ }^{98}$, Alessandra Vie ${ }^{99}$, Valeria Pensotti ${ }^{100,101}$, Stefania Tommasi ${ }^{102}$, Paolo Peterlongo ${ }^{100}$, Jeffrey N. Weitzel ${ }^{103}$, Ana Osorio ${ }^{104,105}$, Javier Benitez ${ }^{105,106,107}$, Lesley McGuffog ${ }^{2}$, Sue Healey ${ }^{108}$, Anne-Marie Gerdes ${ }^{109}$, Bent Ejlertsen ${ }^{110}$, Thomas V. O. Hansen ${ }^{111}$, Linda Steele ${ }^{5}$, Yuan Chun Ding ${ }^{5}$, Nadine Tung ${ }^{112}$, Ramunas Janavicius ${ }^{113}$, David E. Goldgar ${ }^{114}$, Saundra S. Buys ${ }^{115}$, Mary B. Daly ${ }^{116}$, Anita Bane ${ }^{117}$, Mary Beth Terry ${ }^{118}$, Esther M. Johnn ${ }^{119}$, Melissa Southey ${ }^{120}$, Douglas F. Easton ${ }^{2}$, Georgia Chenevix-Trench ${ }^{108}$, Antonis C. Antoniou ${ }^{2+}$ and Laura Ottini ${ }^{1 *+}$

\footnotetext{
*Correspondence: laura.ottini@uniroma1.it

${ }^{\dagger}$ Equal contributors

'Department of Molecular Medicine, Sapienza University of Rome, Viale Regina Elena, 324, 00161 Rome, Italy

Full list of author information is available at the end of the article
} 


\begin{abstract}
Background: BRCA1 and, more commonly, BRCA2 mutations are associated with increased risk of male breast cancer (MBC). However, only a paucity of data exists on the pathology of breast cancers (BCs) in men with BRCA1/2 mutations. Using the largest available dataset, we determined whether MBCs arising in BRCA1/2 mutation carriers display specific pathologic features and whether these features differ from those of BRCA1/2 female BCs (FBCs).

Methods: We characterised the pathologic features of 419 BRCA1/2 MBCs and, using logistic regression analysis, contrasted those with data from 9675 BRCA1/2 FBCs and with population-based data from 6351 MBCs in the Surveillance, Epidemiology, and End Results (SEER) database.

Results: Among BRCA2 MBCs, grade significantly decreased with increasing age at diagnosis $(P=0.005)$. Compared with BRCA2 FBCs, BRCA2 MBCs were of significantly higher stage $\left(P\right.$ for trend $\left.=2 \times 10^{-5}\right)$ and higher grade ( $P$ for trend $=0.005$ ) and were more likely to be oestrogen receptor-positive [odds ratio (OR) 10.59; $95 \%$ confidence interval (CI) 5.15-21.80] and progesterone receptor-positive (OR 5.04; $95 \% \mathrm{Cl} 3.17-8.04$ ). With the exception of grade, similar patterns of associations emerged when we compared BRCA1 MBCs and FBCs. BRCA2 MBCs also presented with higher grade than MBCs from the SEER database ( $P$ for trend $=4 \times 10^{-12}$ ).

Conclusions: On the basis of the largest series analysed to date, our results show that BRCA1/2 MBCs display distinct pathologic characteristics compared with BRCA1/2 FBCs, and we identified a specific BRCA2-associated MBC phenotype characterised by a variable suggesting greater biological aggressiveness (i.e., high histologic grade). These findings could lead to the development of gender-specific risk prediction models and guide clinical strategies appropriate for MBC management.
\end{abstract}

Keywords: Male breast cancer, BRCA1/2, Pathology, Histologic grade, Genotype-phenotype correlations

\section{Background}

Male breast cancer $(\mathrm{MBC})$ is a rare disease. It accounts for less than $1 \%$ of all breast cancers and less than $1 \%$ of all cancers in men. The annual incidence is estimated at about 1 per 100,000 men worldwide [1], and lifetime risk is less than 1 in 1000. Incidence rates for MBC increase linearly and steadily with age, with the mean age at diagnosis being between 60 and 70 years [2]. Family history of breast cancer is an important risk factor for developing $\mathrm{MBC}$, suggesting the importance of genetic factors in MBC susceptibility [3, 4]. Mutations in the two major high-penetrance breast cancer genes, $B R C A 1$ (breast cancer 1 , early onset gene) and predominantly $B R C A 2$ (breast cancer 2, early onset gene), account for approximately $10 \%$ of MBCs outside populations with $B R C A$ founder mutations [5]. The lifetime risk of developing $\mathrm{MBC}$ has been estimated to be in the range of 1-5\% for $B R C A 1$ and $5-10 \%$ for $B R C A 2$ mutation carriers, compared with a risk of $0.1 \%$ in the general population [6-9].

$\mathrm{MBC}$ is recognised as being a hormone-dependent malignancy, and it is widely accepted as an oestrogendriven disease, specifically related to hyperestrogenism [10]. In the general population, $\mathrm{MBC}$ is similar to lateonset, post-menopausal, oestrogen receptor-/progesterone receptor-positive $(\mathrm{ER}+/ \mathrm{PR}+)$ female breast cancer $(\mathrm{FBC})$. However, compared with $\mathrm{FBC}, \mathrm{MBC}$ has been reported to occur later in life, present at a higher stage and display lower histologic grade, with a higher proportion of ER+ and PR+ tumours [11].
There is increasing evidence suggesting that MBC may be a group of molecularly and clinically heterogeneous malignancies which differ from those seen in women [12]. It is well known that breast cancer in women is a heterogeneous disease. Breast cancers arising in female $B R C A 1$ mutation carriers display characteristic pathologic features, including distinct morphology (i.e., carcinomas with medullary features) and a triple-negative phenotype [i.e., ER-, PR-, human epidermal growth factor receptor 2-negative (HER2-)] in the majority. In contrast, $B R C A 2$ breast tumours are a more heterogeneous group, being broadly similar to non-BRCA-associated breast tumours, which more closely resemble post-menopausal FBCs, although with a tendency to be of high grade and HER2- [13].

Current knowledge of the pathologic characteristics of breast cancers arising in male BRCA1/2 mutation carriers is limited, owing to the small number of carriers included in individual studies [14-17]. In a study including 50 male $B R C A 1 / 2$ mutation carriers, it was suggested that $B R C A 2 \mathrm{MBCs}$ may represent a subgroup of tumours with a peculiar phenotype not identified in FBC and characterised by an aggressive biological behaviour [16]. Furthermore, in a study including 28 male $B R C A 1 / 2$ mutation carriers, a possible $B R C A 2$ phenotype characterised by micropapillary histology was suggested [17]. In other, smaller studies, BRCA2 MBCs were associated with younger age at diagnosis and positive lymph node status $[14,15]$. 
In this study, we report pathology data characteristics of 419 BRCA1/2 MBCs derived from the Consortium of Investigators of Modifiers of BRCA1/2 (CIMBA), who conducted the largest study of its kind to date. The main objective of our study was to characterise the pathologic features of $B R C A 1 / 2 \mathrm{MBCs}$ and contrast those with the characteristics of $B R C A 1 / 2 \mathrm{FBCs}$, as well as with $\mathrm{MBCs}$ in the general population.

\section{Methods}

\section{CIMBA study participants}

CIMBA collects data on male and female BRCA1 or $B R C A 2$ pathogenic mutation carriers older than 18 years of age, with the majority recruited through cancer genetics clinics [18]. CIMBA data were submitted by 55 study groups in 24 countries based in Europe, North America and Australia. Pathology data from MBC cases for the present analysis were collected by 35 study groups (Additional file 1). Key variables collected for all CIMBA patients include year of birth, age at cancer diagnosis (breast, ovarian or prostate cancers), age at last observation, family membership, race and/or ethnicity and information on applicable prophylactic surgeries. This work was restricted to male and female mutation carriers who had been diagnosed with breast cancer and were of self-reported European ancestry. The number of male mutation carriers of non-European ancestry ( $2 B R C A 1$ and $20 B R C A 2)$ was too small to allow a meaningful analysis. These subjects were excluded from the analysis.

A signed informed consent form was obtained from study participants. All participating studies were approved by local ethical review committees (Additional file 2).

\section{Tumour pathology data}

$\mathrm{MBC}$ pathology data were obtained from a range of sources, namely medical, pathology or tumour registry records and immunohistochemical staining and/or scoring of tissue microarrays (TMAs) (Additional file 3). The data included information on ER, PR and HER2 status; morphological subtype; lymph node involvement; TNM (tumour, node, metastasis) staging; and histologic grade. For ER, PR and HER2, status was classified as negative or positive. The vast majority of centres employed a cut-off of either $\geq 10 \%$ or $\geq 1 \%$ of tumour nuclei staining positive to define ER/PR receptor positivity, which was not centrally reclassified, owing to the low proportion of records with supporting staining data (Additional file 3). HER2 status was determined using immunohistochemistry (IHC) to detect strong complete membrane staining (with $3+$ considered positive) with in situ hybridisation to detect HER2 gene amplification in equivocal cases. Consistency checks were performed to validate receptor data against supplementary scoring information when provided. Central pathology review was not performed.
Each carcinoma was assigned to a morphologic subgroup (ductal, lobular, medullary, other), which was confirmed using the World Health Organisation International Classification of Diseases 0 code for the classification of tumour type when present. Lymph node status, along with the number of nodes showing metastatic carcinoma, was provided when available. Staging data were based on the AJCC Cancer Staging Manual, Sixth Edition [19], with data provided on overall stage and its major attributes (primary tumour size, regional lymph node involvement and presence of distant metastasis). Histologic grade was determined by local pathologists using modifications of the Scarff-Bloom-Richardson histological grading system as grade 1, 2 or 3. Pathology data for FBCs included in the study are described in detail elsewhere [13].

\section{SEER data}

We obtained MBC pathology data from the SEER 18 Registries Database for cases diagnosed from 1973 to 2011 [20]. For this study, we selected only male Caucasian cases diagnosed with invasive breast cancer. For SEER cases, pathology characteristics included age at diagnosis; morphologic subgroup; tumour grade; lymph node status; adjusted stage based on the AJCC Cancer Staging Manual, Sixth Edition [19]; ER, PR and HER2 status. Tumour grade was classified as grade 1 (well differentiated), grade 2 (moderately differentiated) or grade 3 (poorly differentiated).

SEER includes unselected MBCs, most of which are of unknown BRCA1/2 mutation status. On the basis of published data $[3,21,22]$, about $10 \%$ of $\mathrm{MBC}$ cases are expected to be due to $B R C A 1$ or $B R C A 2$ mutations.

\section{Statistical methods}

Logistic regression was used to assess the association between pathologic characteristics and male $B R C A 1 / 2$ mutation carrier status, as well as to compare pathologic characteristics with data from female $B R C A 1 / 2$ mutation carriers and from male breast tumours arising in the general population using SEER data. In the logistic regression analysis, each pathologic characteristic was treated as the explanatory variable. The outcome variables were $B R C A$ mutation status (BRCA1/BRCA2), sex (female/male) and carrier status (general population/BRCA1 mutation carrier and general population/BRCA2 mutation carrier), with the first term used as the reference group. For assessment of continuous or ordered variables, such as age at diagnosis, stage and grade, tests for trend were also performed.

Analyses within CIMBA data were adjusted for age at diagnosis and country of origin, whereas comparisons between CIMBA and SEER data were adjusted only for age at diagnosis. In addition, an adjustment for calendar year of diagnosis was included in all analyses, based on 
the following groupings: up to 1990, 1991-2000 and after 2000. A robust variance approach was used to allow for dependencies between related individuals. All analyses were carried out using Stata v13 software (StataCorp, College Station, TX, USA).

\section{Results}

Pathologic characteristics of $\mathrm{MBC}$ in $B R C A 1$ and $B R C A 2$ mutation carriers

Information was available for $419 \mathrm{MBC}$ cases, including $375 B R C A 2$ and 44 BRCA1 mutation carriers (Additional file 1). Median age at MBC diagnosis was 62 years [interquartile range (IQR) 16] for BRCA2 mutation carriers and 62 years (IQR 18) for BRCA1 mutation carriers.

The analysis was restricted to carriers diagnosed with invasive breast cancer (326 BRCA2 and 40 BRCA1) (Additional file 4). The majority of tumours were invasive ductal carcinoma in both BRCA2 (95.1\%) and BRCA1 (100\%) carriers. Among tumours with data on stage and grade, the majority of $B R C A 2$ mutation carriers presented with stage 2 disease (47\%) and tumours of histologic grade 3 (56.7\%), whereas the majority of $B R C A 1$ mutation carriers presented with stage 3-4 disease (42.9\%) and histologic grade 3 tumours (69.2\%). Among tumours with ER, PR and HER2 data, $96.7 \%$ were ER+, $86.8 \%$ were $\mathrm{PR}+$ and $83.4 \%$ were HER2- in BRCA2 mutation carriers, vs. $90.3 \% \mathrm{ER}+, 78.6 \% \mathrm{PR}+$ and $89.5 \% \mathrm{HER} 2-$ in BRCA1 mutation carriers.

Age at diagnosis was inversely associated with grade in $B R C A 2$ mutation carriers (grade $1 / 2$ vs. grade $3, P=0.005$ ), with no evidence for differences in ER, PR and HER2 distributions by age (test for differences $P>0.05$ for all) (Fig. 1). Furthermore, there was no evidence of association between grade and ER or PR status ( $P$ values for trend $=0.50$ and 0.78 , respectively). For $B R C A 1$ mutation carriers, no differences in age-specific proportions of tumours by grade or ER, PR and HER2 status were observed, but their numbers were small (data not shown).

When we compared the pathologic characteristics of $\mathrm{MBC}$ in BRCA1 and BRCA2 mutation carriers, we observed no statistically significant differences. However, tumours in BRCA1 mutation carriers were more likely to

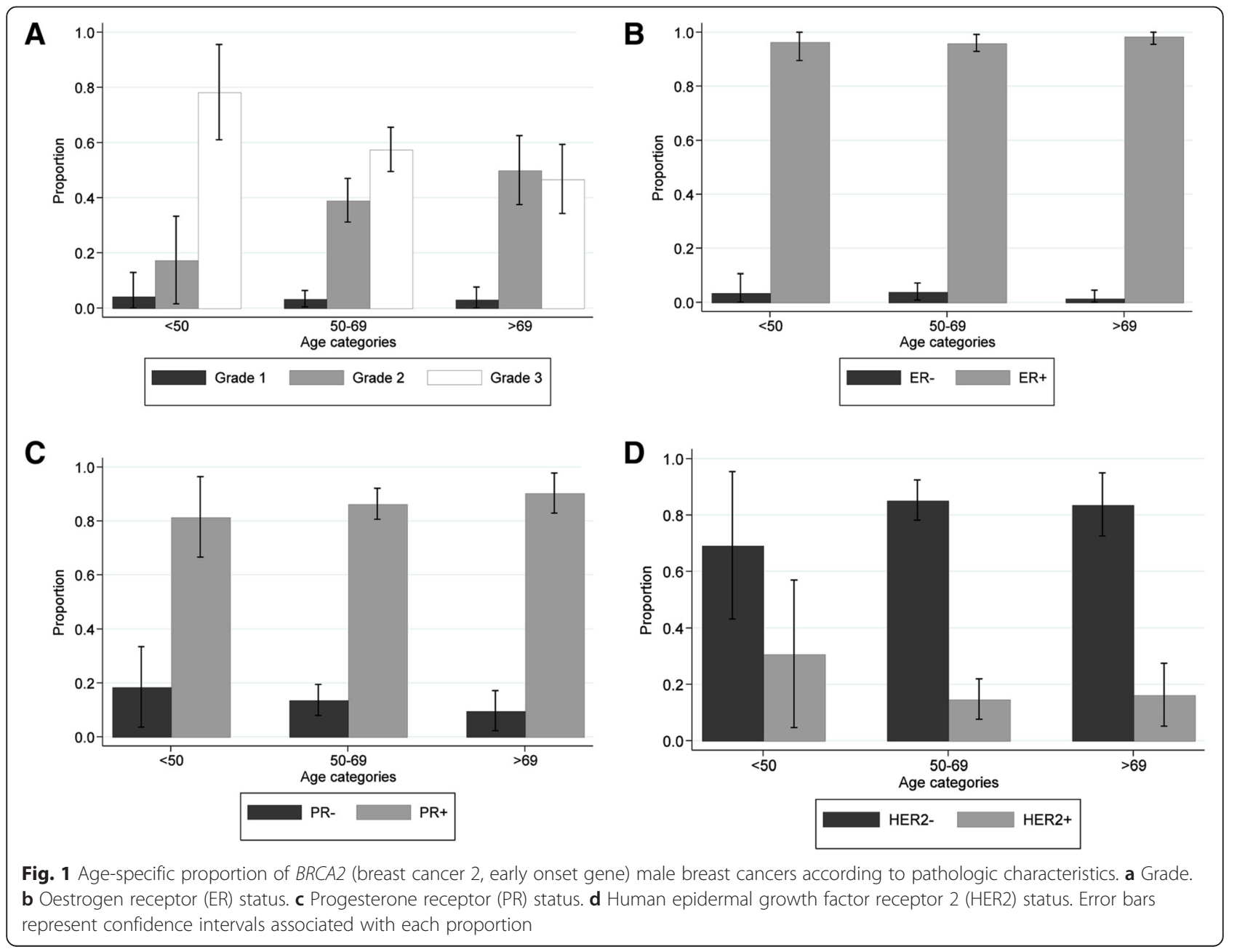


present with more advanced stage ( $42.9 \%$ vs. $23.5 \%, P$ for trend $=0.11)$ and were more frequently ER- $(9.7 \%$ vs. $3.3 \%, P=0.17)$ and PR- $(21.4 \%$ vs. $13.2 \%, P=0.27)$ than tumours in BRCA2 mutation carriers (Additional file 4).

Characterisation of BRCA2 MBCs: comparison with BRCA2 $\mathrm{FBC}$ and with $\mathrm{MBC}$ in the general population

We evaluated possible pathologic differences between invasive breast cancers arising in male and female $B R C A 2$ mutation carriers by comparing available data from female mutation carriers with breast cancer in the CIMBA dataset. Data from 3750 country-matched female BRCA2 mutation carriers diagnosed with invasive breast cancer were included in this analysis (Table 1). The results revealed that there were significantly fewer invasive lobular carcinomas among male $B R C A 2$ mutation carriers than among female $B R C A 2$ mutation carriers [odds ratio (OR) 0.14, $95 \%$ confidence interval (CI) 0.05-0.43]. In addition,

Table 1 Pathology of invasive BRCA2 female and male breast tumours and ORs in predicting male BRCA2 mutation carrier status

\begin{tabular}{|c|c|c|c|c|c|c|}
\hline & \multicolumn{2}{|l|}{ Females } & \multicolumn{2}{|l|}{ Males } & \multirow[t]{2}{*}{ Unadjusted OR (95 \% Cl) } & \multirow[t]{2}{*}{ Adjusted $\mathrm{OR}^{\mathrm{a}}(95 \% \mathrm{Cl})$} \\
\hline & Number & Percent & Number & Percent & & \\
\hline Total $^{b}$ & 3750 & & 326 & & & \\
\hline \multicolumn{7}{|l|}{ Morphology } \\
\hline Ductal carcinoma & 2693 & 83.6 & 253 & 95.1 & Reference & Reference \\
\hline Lobular carcinoma & 276 & 8.6 & 4 & 1.5 & $0.15(0.06-0.41)$ & $0.14(0.05-0.43)$ \\
\hline Medullary carcinoma & 60 & 1.9 & 2 & 0.8 & $0.35(0.09-1.46)$ & $0.46(0.10-2.11)$ \\
\hline Other & 193 & 6.0 & 7 & 2.6 & $0.39(0.18-0.83)$ & $0.54(0.24-1.23)$ \\
\hline \multicolumn{7}{|l|}{ TNM stage } \\
\hline $0-1$ & 560 & 40.2 & 44 & 29.5 & Reference & Reference \\
\hline 2 & 629 & 45.1 & 70 & 47.0 & $1.42(0.95-2.10)$ & $1.97(1.20-3.23)$ \\
\hline $3-4$ & 205 & 14.7 & 35 & 23.5 & $2.17(1.37-3.44)$ & $3.55(1.96-6.44)$ \\
\hline \multicolumn{7}{|l|}{ Histologic grade } \\
\hline Grade 1 & 149 & 5.9 & 8 & 3.5 & Reference & Reference \\
\hline Grade 2 & 1057 & 41.7 & 92 & 39.8 & $1.62(0.77-3.41)$ & $1.88(0.76-4.67)$ \\
\hline Grade 3 & 1329 & 52.4 & 131 & 56.7 & $1.84(0.88-3.83$ & $2.66(1.08-6.55)$ \\
\hline \multicolumn{7}{|l|}{ Lymph node status } \\
\hline Negative & 1398 & 52.4 & 123 & 50.2 & Reference & Reference \\
\hline Positive & 1270 & 47.6 & 122 & 49.8 & $1.09(0.84-1.43)$ & $1.55(1.12-2.14)$ \\
\hline \multicolumn{7}{|l|}{ ER status } \\
\hline Negative & 650 & 22.7 & 8 & 3.3 & Reference & Reference \\
\hline Positive & 2211 & 77.3 & 236 & 96.7 & 8.67 (4.26-17.66) & $10.59(5.15-21.80)$ \\
\hline \multicolumn{7}{|l|}{ PR status } \\
\hline Negative & 892 & 35.0 & 30 & 13.2 & Reference & Reference \\
\hline Positive & 1654 & 65.0 & 198 & 86.8 & $3.56(2.41-5.26)$ & $5.04(3.17-8.04)$ \\
\hline \multicolumn{7}{|l|}{ HER2 status } \\
\hline Negative & 1404 & 85.9 & 126 & 83.4 & Reference & Reference \\
\hline Positive & 230 & 14.1 & 25 & 16.6 & $1.21(0.77-1.90)$ & $1.22(0.70-2.11)$ \\
\hline \multicolumn{7}{|l|}{ Subtypes } \\
\hline ER+ and/or PR+, HER2- & 1112 & 69.8 & 118 & 81.9 & Reference & Reference \\
\hline ER+ and/or PR+, HER2+ & 182 & 11.4 & 22 & 15.3 & $1.14(0.70-1.84)$ & $1.18(0.65-2.13)$ \\
\hline $\mathrm{ER}-, \mathrm{PR}-, \mathrm{HER} 2+$ & 40 & 2.5 & 2 & 1.4 & $0.47(0.11-1.98)$ & $0.42(0.09-1.98)$ \\
\hline Triple-negative (ER-, PR-, HER2-) & 260 & 16.3 & 2 & 1.4 & $0.07(0.02-0.30)$ & $0.05(0.01-0.22)$ \\
\hline ER+ and/or PR+, HER2- vs. others & & & & & $0.51(0.33-0.79)$ & $0.42(0.25-0.70)$ \\
\hline
\end{tabular}

$B R C A 2$ breast cancer 2, early onset gene, $C$ confidence interval, ER oestrogen receptor, HER2 human epidermal growth factor receptor 2 , OR odds ratio, $P R$ progesterone receptor, TNM tumour, node, metastasis

Significant results are indicated by boldface type

${ }^{a}$ Analyses adjusted for country, age at diagnosis and calendar year of diagnosis

${ }^{b}$ Some data for each pathologic feature are not available 
compared with BRCA2 FBCs, BRCA2 MBCs were of significantly higher stage $\left(P\right.$ for trend $\left.=2.14 \times 10^{-5}\right)$ and higher grade $(P$ for trend $=0.005)$, presented more frequently with lymph node involvement (OR 1.55, 95 \% CI 1.12-2.14) and were more likely to be ER+ (OR 10.59, 95 \% CI 5.15-21.80), PR+ (OR 5.04; 95 \% CI 3.17-8.04) and non-triple-negative (OR 0.05, $95 \%$ CI 0.01-0.22). Associations with stage and nodal, ER and PR status remained significant after adjustment for grade.
We then compared pathologic features of $\mathrm{MBC}$ arising in BRCA2 mutation carriers with characteristics of MBC in the general U.S. population as represented by SEER. We extracted pathology data of 6351 men with invasive breast cancer from the SEER 18 database. There were no statistically significant differences in pathology characteristics between $\mathrm{MBCs}$ arising in BRCA2 mutation carriers and those arising in the general population, with the exception of grade and lymph node status (Table 2).

Table 2 Pathology of invasive MBCs in the general population from SEER and BRCA2 MBCs and ORs in predicting male BRCA2 mutation carrier status

\begin{tabular}{|c|c|c|c|c|c|c|}
\hline & \multicolumn{2}{|l|}{ SEER } & \multicolumn{2}{|c|}{ BRCA2 carriers } & \multirow[t]{2}{*}{ Unadjusted OR (95 \% Cl) } & \multirow[t]{2}{*}{ Adjusted $\mathrm{OR}^{\mathrm{a}}(95 \% \mathrm{Cl})$} \\
\hline & Number & Percent & Number & Percent & & \\
\hline$\overline{\text { Total }^{b}}$ & 6351 & & 326 & & & \\
\hline \multicolumn{7}{|l|}{ Morphology } \\
\hline Ductal carcinoma & 5265 & 86.2 & 253 & 95.1 & Reference & Reference \\
\hline Lobular carcinoma & 82 & 1.5 & 4 & 1.5 & $1.02(0.37-2.79)$ & $1.00(0.36-2.74)$ \\
\hline Medullary Carcinoma & 16 & 0.3 & 2 & 0.8 & $2.60(0.59-11.38)$ & $2.34(0.52-10.39)$ \\
\hline \multicolumn{7}{|l|}{ TNM stage } \\
\hline $0-1$ & 1699 & 34.9 & 44 & 29.5 & Reference & Reference \\
\hline 2 & 1990 & 40.9 & 70 & 47.0 & $1.36(0.93 .-1.99)$ & $1.37(0.93-2.01)$ \\
\hline $3-4$ & 1181 & 24.2 & 35 & 23.5 & $1.14(0.73-1.77)$ & $1.11(0.72-1.73)$ \\
\hline \multicolumn{7}{|l|}{ Histologic grade } \\
\hline Grade 1 & 632 & 12.9 & 8 & 3.5 & Reference & Reference \\
\hline Grade 2 & 2432 & 49.7 & 92 & 39.8 & $2.99(1.44-6.19)$ & $2.98(1.44-6.19)$ \\
\hline Grade 3 & 1834 & 37.4 & 131 & 56.7 & $5.64(2.75-11.60)$ & $5.53(2.69-11.39)$ \\
\hline \multicolumn{7}{|l|}{ Lymph node status } \\
\hline Negative & 2773 & 58.0 & 123 & 50.2 & Reference & Reference \\
\hline Positive & 2009 & 42.0 & 122 & 49.8 & $1.37(1.05-1.78)$ & $1.28(0.98-1.67)$ \\
\hline \multicolumn{7}{|l|}{ ER status } \\
\hline Negative & 229 & 5.3 & 8 & 3.3 & Reference & Reference \\
\hline Positive & 4064 & 94.7 & 236 & 96.7 & $1.66(0.81-3.41)$ & $1.95(0.93-4.06)$ \\
\hline \multicolumn{7}{|l|}{ PR status } \\
\hline Negative & 627 & 15.0 & 30 & 13.2 & Reference & Reference \\
\hline Positive & 3562 & 85.0 & 198 & 86.8 & $1.16(0.79-1.72)$ & $1.30(0.88-1.92)$ \\
\hline \multicolumn{7}{|l|}{ HER2 status } \\
\hline Negative & 627 & 87.8 & 126 & 83.4 & Reference & Reference \\
\hline Positive & 87 & 12.2 & 25 & 16.6 & $1.43(0.88-2.32)$ & $1.30(0.79-2.13)$ \\
\hline \multicolumn{7}{|l|}{ Subtypes } \\
\hline ER+ and/or PR+, HER2- & 608 & 87.5 & 118 & 81.9 & Reference & Reference \\
\hline ER+ and/or PR+, HER2+ & 80 & 11.5 & 22 & 15.3 & $1.42(0.85-2.36)$ & $1.28(0.76-2.17)$ \\
\hline ER-, PR-, HER2+ & 7 & 1.0 & 2 & 1.4 & $1.47(0.30-7.18)$ & $1.09(0.22-5.45)$ \\
\hline Triple-negative (ER-, PR-, HER2-) & 0 & 0.0 & 2 & 1.4 & - & - \\
\hline ER+ and/or PR+, HER2- vs. others & & & & & $1.54(0.95-2.49)$ & $1.38(0.84-2.27)$ \\
\hline
\end{tabular}

$B R C A 2$ breast cancer 2, early onset gene, $C l$ confidence interval, ER oestrogen receptor, HER2 human epidermal growth factor receptor 2, OR odds ratio, $P R$ progesterone receptor, TNM tumour, node, metastasis

Significant results are indicated by boldface type

${ }^{a}$ Analyses adjusted for age at diagnosis and calendar year of diagnosis

${ }^{b}$ Some data for each pathologic feature are not available 
Male $B R C A 2$ mutation carriers more frequently had grades 2 and 3 tumours than grade 1 tumours, as compared with $\mathrm{MBC}$ cases from the general population (grade 2 vs. grade 1 OR 2.98, $95 \%$ CI 1.44-6.19; grade 3 vs. grade 1 OR 5.53, $95 \%$ CI 2.69-11.39; $P$ for trend $=4.52 \times 10^{-12}$ ). Moreover, BRCA2 mutation carriers presented more frequently with lymph node involvement than $\mathrm{MBC}$ cases from the general population, a difference that was not significant when adjusted for age at diagnosis and/or grade.

\section{Characterisation of BRCA1 MBCs: comparison with BRCA1 $\mathrm{FBC}$ and with $\mathrm{MBC}$ in the general population}

A total of 5925 country-matched female BRCA1 mutation carriers diagnosed with invasive breast cancer were compared with our BRCA1 MBC series, which revealed that $\mathrm{MBCs}$ were of significantly higher stage (stage $3-4$ vs. stage 1 OR 17.59, $95 \%$ CI 3.47-89.03; $P$ for trend $=0.001$ ) and presented more frequently with lymph node involvement (OR 2.19, 95 \% CI 1.03-4.65) than FBCs in BRCA1 mutation carriers (Additional file 5). The association with stage remained significant after adjusting for ER and PR status. Moreover, BRCA1 MBCs were more likely to be $\mathrm{ER}+$ (OR 20.22, 95 \% CI 5.91-69.17), PR+ (OR 13.76, 95 $\%$ CI 5.31-35.67) and non-triple-negative (OR 0.03, $95 \%$ CI $0.00-0.25)$. The associations with ER and PR status remained significant after adjustment for stage. There was no statistically significant difference in the distribution of histologic grade among male and female BRCA1 breast cancers.

The comparison between MBCs arising in BRCA1 mutation carriers with those of $6351 \mathrm{MBCs}$ from the SEER database showed no significant differences in pathologic characteristics (Additional file 6). However, BRCA1 male breast tumours trended toward higher grade compared with those in the general population $(P$ for trend $=0.003$ ).

\section{Discussion}

To date, most of the available knowledge on $\mathrm{MBC}$ is based on $\mathrm{MBC}$ arising in the general population, whose $B R C A 1 / 2$ mutation status is largely unknown. In this study, we sought to determine whether $\mathrm{MBC}$ arising in $B R C A 1$ and BRCA2 mutation carriers displayed specific pathologic characteristics. We used data on $419 \mathrm{MBCs}$ with $B R C A 1$ and $B R C A 2$ mutations from an international consortium (CIMBA). The CIMBA series represents the largest collection of MBCs arising in BRCA1 and BRCA2 mutation carriers to date. In our series, the majority of $\mathrm{MBC}$ cases (375 of 419, $89.5 \%$ ) were BRCA2 mutation carriers, a finding which corroborates prior, smaller studies.

In this study, we conducted the first comparison of the pathologic features of breast cancer arising in male and female $B R C A 1 / 2$ mutation carriers, taking advantage of the previously collected pathology data from female BRCA1/2 mutation carriers assembled by CIMBA [13].
We found that breast cancer in male $B R C A 2$ mutation carriers was of significantly higher stage and histologic grade, and was more frequently $\mathrm{ER}+$ and $\mathrm{PR}+$, than breast cancer in female BRCA2 mutation carriers. Advanced stage disease at breast cancer diagnosis is more frequently observed in men than in women [23]. In general, this is thought to reflect diagnostic delay in a population unaware of its risk and (appropriately) not encouraged to undergo routine breast cancer screening. Furthermore, although breast cancer primaries in men tend to be slightly smaller than those in women when they are first diagnosed, they more often have locoregional metastasis at presentation. Indeed, we found that male BRCA2 mutation carriers presented more frequently with lymph node involvement than breast cancer in female mutation carriers.

It is known that $\mathrm{MBC}$ presents with lower histologic grade tumours than FBC in the general population [11]. In contrast, in the present study, we showed that MBC associated with $B R C A 2$ mutations presents with higher histologic grade than both breast cancer in female $B R C A 2$ mutation carriers and $\mathrm{MBC}$ in the general population from SEER.

We observed that the majority of $B R C A 2 \mathrm{MBCs}$ are of grades 2 and 3 . However, grade 3 tumours were more frequent among male $B R C A 2$ mutation carriers diagnosed at younger ages (younger than age 50 years) than among those diagnosed at older ages, whereas grade 2 tumours showed an inverse trend. Age-specific proportions of MBCs stratified by grade show that grade 3 significantly decreased with increasing age in male $B R C A 2$ mutation carriers. These results may indicate that young male $B R C A 2$ mutation carriers could be susceptible to more aggressive (i.e., high-grade) breast cancer. Differences in grade among male breast carcinomas by age may be an indicator of a biologic complexity in MBC, as suggested in FBC [24].

In a previous, single-country case series, $\mathrm{MBCs}$ associated with $B R C A 2$ mutations were found to be of higher grade than non-BRCA2 MBC [16]. In the present study, we confirmed this association in a large, multicentre series and showed that this association was age-specific. The identification of a specific $B R C A 2$-associated phenotype suggestive of an aggressive behaviour might define a subset of $\mathrm{MBC}$ patients (i.e., patients with high-grade breast tumours and with young age at diagnosis) who may particularly benefit from adjuvant chemotherapy $[2,25]$.

We also showed that high-grade breast tumours were more likely to arise in male than in female $B R C A 2$ mutation carriers, indicating that $B R C A 2$ mutations might be associated with different breast cancer phenotypes in men and in women. It has been suggested that high grade is a surrogate for proliferation, and, although the evidence is conflicting, this may add to the understanding of the molecular differences of $\mathrm{MBC}$ and $\mathrm{FBC}$. 
MBC is recognised as being primarily a hormonedependent malignancy, and, in general, $\mathrm{MBC}$ is described as being more frequently $\mathrm{ER}+$ and $\mathrm{PR}+$ than $\mathrm{FBC}[10,11$, 23]. In the present study, we showed that $B R C A 2 \mathrm{MBCs}$ are more likely than $B R C A 2 \mathrm{FBCs}$ to be $\mathrm{ER}+$ and $\mathrm{PR}+$, thus suggesting that susceptibility to hereditary breast cancer may be influenced by differences in hormonal background between male and female $B R C A 2$ mutation carriers.

Invasive lobular carcinomas are very rare in men, accounting for only about $2 \%$ of all MBCs [23, 26]. We also found significantly fewer lobular carcinomas among male than female BRCA2 mutation carriers. However, it is worth noting that breast cancers in female $B R C A 2$ mutation carriers frequently show a lobular morphology [13], thus suggesting differences in the pathogenic mechanisms of male and female $B R C A 2$ breast cancer.

The number of $\mathrm{MBC}$ cases with $B R C A 1$ mutations in our datasets was much smaller than the number of BRCA2 mutation carriers, and our results in this subset of patients should therefore be interpreted with caution. We found that BRCA1 MBC cases were of significantly higher stage, and more frequently $\mathrm{ER}+$ and $\mathrm{PR}+$, than BRCA1 FBCs. Despite the small sample size, our results suggest that hormone receptor pathways also are a driving force in BRCA1 MBC. It is well known that most of the breast tumours arising in female $B R C A 1$ mutation carriers tend to be ER- and PR-, with a small percentage being ER $+[13,27,28]$. Given that both ER- and ER+ BRCA1 breast cancers seem to originate from a common luminal progenitor cell population, it has been suggested that ER status of breast cancer occurring in BRCA1 mutation carriers may be under control of different molecular mechanisms [29]. The finding that MBCs associated with $B R C A 1$ mutations are frequently ER+ suggests that the hormonal milieu may be a mechanism controlling ER status in BRCA1 tumours. The different hormonal background between males and females and the absence of hormone exposures related to reproductive history in males as compared with $\mathrm{FBC}$ may also influence biologic and molecular mechanisms underlying the pathologic differences between $\mathrm{MBC}$ and FBC. Following the findings in the present study, future studies are warranted which focus on the comprehensive somatic and molecular profiling of $\mathrm{MBC}$ and $\mathrm{FBC}$ in mutation carriers. Such studies could provide new insights into the complex nature of the origin and evolution of $\mathrm{MBC}$ and $\mathrm{FBC}$.

Interestingly, we found no statistically significant differences in the pathologic characteristics between MBCs in BRCA1/2 mutation carriers and those in the general population, with the exception of histologic grade. Male BRCA2 mutation carriers more frequently have grade $2 / 3$ vs. grade 1 tumours, compared with the large, unselected population of MBC cases from SEER. A similar trend also was observed for BRCA1 mutation carriers. These findings suggest that, although $\mathrm{MBCs}$ arising in male $B R C A 1 / 2$ mutation carriers seem to be very similar to MBCs arising in the general population, according to morphologic and immunophenotypic features, they represent a subgroup characterised by aggressive biology.

The importance of histologic grade as a prognostic factor in breast cancer has been ascertained in FBC [30]. Recent data indicate that high-grade tumours are associated with shorter disease-free survival and overall survival rates in MBC patients [25]. Thus, on the basis of our results, we can suggest that $B R C A 2 \mathrm{MBC}$ may display an aggressive phenotype and possibly a more unfavourable prognosis. This is a question in need of additional survival data that we are planning to collect within CIMBA.

In this study, tumour pathology data were collected through several mechanisms, including medical records, pathology reports and TMAs. Given the global distribution of CIMBA study sites, central pathology review was not feasible. Laboratory methods for tissue preparation, IHC, biochemical assays, scoring systems and data interpretation vary widely (Additional file 3), and misclassifications cannot be excluded. Unfortunately, details of hormone receptor scoring for all mutation carriers were not available to standardise definitions across centres. However, data collected by CIMBA are more representative of typical assessment of pathology conducted in routine practice, and the distributions of hormone receptors' status across different study centres and countries in CIMBA were generally consistent. There was some variation in the distribution of some variables, including ER status, probably due to changing assay thresholds and detection methods over time and from country to country. Therefore, adjustments based on calendar year of diagnosis and country of origin were included for all analyses. Missing data for some variables, including HER2 status, and the very small number of male BRCA1 mutation carriers in the study may have impacted the statistical power to detect associations.

CIMBA collects data only on BRCA1 and BRCA2 mutation carriers. Therefore, to compare the tumour characteristics of $\mathrm{MBC}$ from the general population, we took advantage of the publicly available SEER data [20]. Although the U.S. SEER program is the largest source of epidemiologic information on the incidence and survival rates of cancer, it includes data from a single country, and this represents a limitation when attempting to generalise our findings to what one would expect in a collaborative international consortium. However, results from this study, based on a large, multicentre series, replicated previous findings of much smaller studies carried out in single populations [14-17], providing some reassurance that our results were not biased by the different selection of cases in SEER and in CIMBA. In addition, SEER includes MBCs that were not screened for $B R C A 1$ and $B R C A 2$ mutations, and it can be expected that about 
$10 \%$ of those cases $[3,21,22]$ may be due to $B R C A 1$ or $B R C A 2$ mutations. In future studies, researchers should aim to compare $B R C A 1 / 2 \mathrm{MBC}$ cases with those known not to have $B R C A 1 / 2$ mutations.

\section{Conclusions}

Analysing the largest series of $B R C A 1$ and $B R C A 2$ breast cancers collected to date from both sexes, we have demonstrated that breast tumours arising in BRCA1 and $B R C A 2$ mutation carriers display pathologic differences between males and females. Thus, our results add to the accumulating evidence that breast cancer may not be the same disease in both genders [12] and suggest that the heritable influence on breast cancer susceptibility may be context-dependent, perhaps influenced by the microenvironment (i.e., a different hormonal milieu in males and females).

Moreover, we identified a specific $B R C A 2$-associated $M B C$ phenotype characterised by higher histologic grade compared with both $B R C A 2 \mathrm{FBC}$ and $\mathrm{MBC}$ from a general population. This raises the possibility that $B R C A 2 \mathrm{MBC}$ may be more aggressive than its sporadic counterpart.

Overall, our findings could lead to the eventual development of clinical strategies appropriate for MBC management, and of gender-specific risk prediction models that might guide more targeted screening and surveillance programs for male mutation carriers.

\section{Additional files}

\section{Additional file 1: Male $B R C A 1$ and $B R C A 2$ mutation carriers by study group/country. (DOCX $21 \mathrm{~kb})$}

Additional file 2: List of local ethics committees that granted approval for the access and use of the data in present study. (DOCX $23 \mathrm{~kb}$ )

Additional file 3: Methods and thresholds used to define the final marker variables for study groups providing MBC cases. (DOCX $20 \mathrm{~kb}$ )

Additional file 4: Pathology of $B R C A 1$ and $B R C A 2$ MBCs and ORs in predicting $B R C A 2$ mutation carrier status. (DOCX $20 \mathrm{~kb}$ )

Additional file 5: Pathology of invasive $B R C A 1$ female and male breast tumours and ORs in predicting male BRCA1 mutation carrier status. (DOCX $19 \mathrm{~kb}$ )

Additional file 6: Pathology of invasive MBCs in the general population from SEER and BRCA1 MBCs and ORs in predicting male BRCA1 mutation carrier status. (DOCX $19 \mathrm{~kb}$ )

\section{Abbreviations \\ BC: breast cancer; BRCA1: breast cancer 1, early onset gene; BRCA2: breast cancer 2, early onset gene; $\mathrm{Cl}$ : confidence interval; CIMBA: Consortium of Investigators of Modifiers of BRCA1/2; ER: oestrogen receptor; FBC: female breast cancer; HER2: human epidermal growth factor receptor 2; IHC: immunohistochemistry; IQR: interquartile range; MBC: male breast cancer; OR: odds ratio; PR: progesterone receptor; SEER: Surveillance, Epidemiology, and End Results; TMA: tissue microarray; TNM: tumour, node, metastasis.}

Competing interests

The authors declare that they have no competing interests.

\section{Authors' contributions}

Conception and design: ACA and LO. Acquisition of data: AMM, SLN, SF, BYK, GM, PJ, DLT, KKZ, NJC, KLN, SMD, TRR, SJR, RLN, OIO, JR, SY, MAC, LS, AB, ISP, MT, UBJ, AET, LS, ILA, GG, PJH, ENI, MHG, PLM, CFS, CR, GK, JV, KO, MR, AL, LJ, EM, LF, MN, PV, FJC, EH, KJR, PS, SK, KConFab, MRT, PP, MM, LM, AA, OTJ, RBB, AJ, JL, Al, MAP, JB, OD, EO, JP, Gl, AK, HEBON, HN, KA, PPS, TC, TVM, BP, KBMC, Cl, $C E, C L, D S, L B, M B, A M, A G, C S, C E, D N, D S, E H, K K, N A, R V, D W, A K G, D G E, D F, J P$, $J A$, LI, RP, RE, SE, EMBRACE, UH, JG, FF, GF, BP, GG, PR, AR, LC, LP, LV, DP, IZ, AS, PR, $S M, B P, M B, B B, A V, V P, S T, P P, J N W, A O, J B, L M G, S H, A G, B E, T V O H, L S, Y C D, N T, R$, $\mathrm{DEG}, \mathrm{SSB}, \mathrm{MBD}, \mathrm{AB}, \mathrm{MBT}, \mathrm{EMJ}, \mathrm{MS}, \mathrm{DFE}$ and GCT. Analysis and interpretation of data: VS, DB, ACA and LO. Writing of the manuscript: VS, DB, AMM, SLN, SF, ACA and LO. All authors read and approved the final manuscript.

\section{Acknowledgements}

BCFR: This work was supported by grant UM1 CA164920 from the National Cancer Institute. The content of this manuscript does not necessarily reflect the views or policies of the National Cancer Institute or any of the collaborating centres in the Breast Cancer Family Registry (BCFR), nor does mention of trade names, commercial products, or organizations imply endorsement by the U.S. government or the BCFR. BCFR-AU: Acknowledge Maggie Angelakos, Judi Maskiell, Gillian Dite and Helen Tsimiklis. BCFR-NY: We thank members and participants in the New York site of the Breast Cancer Family Registry for their contributions to the study. BCFR-ON: We thank members and participants in the Ontario Familial Breast Cancer Registry for their contributions to the study. BFBOCC-LT is partly supported by Research Council of Lithuania grant LIG-07/2012. We acknowledge Vilius Rudaitis and Laimonas Griškevičius. BIDMC is supported by the Breast Cancer Research Foundation. BRICOH: SLN was partially supported by the Morris and Horowitz Families Endowed Professorship. CBCS: This work was supported by the NEYE Foundation. CNIO: This work was partially supported by Spanish Association against Cancer (AECC08), RTICC 06/0020/1060, FISPI08/1120, Mutua Madrileña Foundation (FMMA) and SAF2010-20493. We thank Alicia Barroso, Rosario Alonso and Guillermo Pita for their assistance. COH-CCGCRN: City of Hope Clinical Cancer Genetics Community Network and the Hereditary Cancer Research Registry, supported in part by award number RC4CA153828 (JNW, principal investigator) from the National Cancer Institute and the Office of the Director, National Institutes of Health. The content is solely the responsibility of the authors and does not necessarily represent the official views of the National Institutes of Health. CONSIT TEAM: Funds from Italian citizens who allocated the $5 \times 1000$ share of their tax payment in support of the Fondazione IRCCS Istituto Nazionale Tumori, according to Italian laws (INT institutional strategic projects ' $5 \times 1000$ ') to SM and from FiorGen Foundation for Pharmacogenomics to LP. LO is supported by AIRC (IG12780). VS is supported by FIRC (triennial fellowship "Mario e Valeria Rindi"). We acknowledge Maria Grazia Tibiletti of the Ospedale di Circolo-Università dell'Insubria, Varese, Italy. CORE: The CIMBA data management and data analysis were supported by Cancer Research UK grants C12292/A11174 and C1287/A10118. SH is supported by an NHMRC program grant (to GCT). ACA is a Cancer Research UK senior cancer research fellow. GCT is an NHMRC senior principal research fellow. DEMOKRITOS: This research has been co-financed by the European Union [European Social Fund (ESF)] and Greek national funds through the Operational Program "Education and Lifelong Learning" of the National Strategic Reference Framework (NSRF) - Research Funding Program of the General Secretariat for Research \& Technology: ARISTEIA. Investing in knowledge society through the European Social Fund. DKFZ: The DKFZ study was supported by the DKFZ. EMBRACE is supported by Cancer Research UK grants C1287/A10118 and C1287/A11990. DGE and Fiona Lalloo are supported by an NIHR grant to the Biomedical Research Centre, Manchester, UK. The investigators at The Institute of Cancer Research and The Royal Marsden NHS Foundation Trust are supported by an $\mathrm{NIHR}$ grant to the Biomedical Research Centre at The Institute of Cancer Research and The Royal Marsden NHS Foundation Trust. RE and Elizabeth Bancroft are supported by Cancer Research UK grant C5047/A8385. RE is also supported by NIHR support of the Biomedical Research Centre at The Institute of Cancer Research and The Royal Marsden NHS Foundation Trust. RE is supported by NIHR support to the Biomedical Research Centre at The Institute of Cancer Research and The Royal Marsden NHS Foundation Trust. FCCC: The authors acknowledge support from The University of Kansas Cancer Center (P30 CA168524) and the Kansas Bioscience Authority Eminent Scholar Program. AKG was funded by 5U01CA113916, R01CA140323, and by the Chancellors Distinguished Chair in Biomedical Sciences Professorship. We thank Ms. Jo Ellen Weaver and Dr. Betsy Bove for their technical support. GC-HBOC: The German 
Consortium of Hereditary Breast and Ovarian Cancer (GC-HBOC) is supported by the German Cancer Aid (grant 110837 to Rita K. Schmutzler) and by the Center for Molecular Medicine Cologne (CMMC). GEMO: The study was supported by the Ligue Nationale Contre le Cancer; the Association "Le cancer du sein, parlons-en!" Award; the Canadian Institutes of Health Research for the "CIHR Team in Familial Risks of Breast Cancer" program and the French National Institute of Cancer (INCa). Genetic Modifiers of Cancer Risk in BRCA1/2 Mutation Carriers (GEMO) study: National Cancer Genetics Network "UNICANCER Genetic Group", France. We thank all the GEMO collaborating groups for their contribution to this study. GEMO Collaborating Centers are Coordinating Centres: Unité Mixte de Génétique Constitutionnelle des Cancers Fréquents, Hospices Civils de Lyon - Centre Léon Bérard, \& Equipe "Génétique du cancer du sein», Centre de Recherche en Cancérologie de Lyon: Olga Sinilnikova ${ }^{\dagger}$, Sylvie Mazoyer, Francesca Damiola, Laure Barjhoux, Carole Verny-Pierre, Mélanie Léone, Nadia Boutry-Kryza, Alain Calender, Sophie Giraud; and Service de Génétique Oncologique, Institut Curie, Paris: Dominique Stoppa-Lyonnet, Marion GauthierVillars, Bruno Buecher, Claude Houdayer, Etienne Rouleau, Lisa Golmard, Agnès Collet, Virginie Moncoutier, Cédrick Lefol, Muriel Belotti, Antoine de Pauw, Camille Elan, Catherine Nogues, Emmanuelle Fourme, Anne-Marie Birot. Institut Gustave Roussy, Villejuif: Brigitte Bressac-de-Paillerets, Olivier Caron, Marine Guillaud-Bataille. Centre Jean Perrin, Clermont-Ferrand: Yves-Jean Bignon, Nancy Uhrhammer. Centre Léon Bérard, Lyon: Christine Lasset, Valérie Bonadona, Sandrine Handallou. Centre François Baclesse, Caen: Agnès Hardouin, Pascaline Berthet, Dominique Vaur, Laurent Castera. Institut Paoli Calmettes, Marseille: Hagay Sobol, Violaine Bourdon, Tetsuro Noguchi, Audrey Remenieras, François Eisinger. CHU Arnaud-de-Villeneuve, Montpellier: Isabelle Coupier, Pascal Pujol. Centre Oscar Lambret, Lille: Jean-Philippe Peyrat, Joëlle Fournier, Françoise Révillion, Philippe Vennin ${ }^{\dagger}$, Claude Adenis. Centre Paul Strauss, Strasbourg: Danièle Muller, Jean-Pierre Fricker. Institut Bergonié, Bordeaux: Emmanuelle Barouk-Simonet, Françoise Bonnet, Virginie Bubien, Nicolas Sevenet, Michel Longy. Institut Claudius Regaud, Toulouse: Christine Toulas, Rosine Guimbaud, Laurence Gladieff, Viviane Feillel. CHU Grenoble: Dominique Leroux, Hélène Dreyfus, Christine Rebischung, Magalie Peysselon. CHU Dijon: Fanny Coron, Laurence Faivre. CHU St-Etienne: Fabienne Prieur, Marine Lebrun, Caroline Kientz. Hôtel Dieu Centre Hospitalier, Chambéry: Sandra Fert Ferrer. Centre Antoine Lacassagne, Nice: Marc Frénay. CHU Limoges: Laurence Vénat-Bouvet. CHU Nantes: Capucine Delnatte. CHU Bretonneau, Tours: Isabelle Mortemousque. Groupe Hospitalier Pitié-Salpétrière, Paris: Florence Coulet, Chrystelle Colas, Florent Soubrier, Mathilde Warcoin. CHU Vandoeuvre-les-Nancy: Johanna Sokolowska, Myriam Bronner. CHU Besançon: Marie-Agnès Collonge-Rame, Alexandre Damette. Creighton University, Omaha, NE, USA: Henry T. Lynch, Carrie L. Snyder. Georgetown University: CI received support from the Non-Therapeutic Subject Registry Shared Resource at Georgetown University ( $\mathrm{NIH} / \mathrm{NCl}$ grant P30-CA051008), the Fisher Center for Familial Cancer Research, and Swing for the Cure. G-FAST: Kim De Leeneer is supported by GOA grant BOF10/GOA/019 (Ghent University) and spearhead financing of Ghent University Hospital. We thank the technical support of Ilse Coene en Brecht Crombez. HCSC was supported by a grant RD12/00369/0006 and 12/00539 from ISCIII (Spain), partially supported by European Regional Development FEDER funds. We acknowledge Alicia Tosar for her technical assistance. HEBCS was financially supported by the Helsinki University Hospital Research Fund, Academy of Finland (266528), the Finnish Cancer Society and the Sigrid Juselius Foundation. HEBCS thanks Taru A. Muranen, Drs. Carl Blomqvist and Kirsimari Aaltonen and RNs Irja Erkkilä and Virpi Palola for their help with the HEBCS data and samples. HEBON is supported by the Dutch Cancer Society grants NKI1998-1854, NKI2004-3088, NKI2007-3756, the Netherlands Organization of Scientific Research grant NWO 91109024, the Pink Ribbon grant 110005 and the BBMRI grant NWO 184.021.007/CP46. HEBON thanks the registration teams of the Comprehensive Cancer Centre Netherlands and Comprehensive Centre South (together the Netherlands Cancer Registry) and PALGA (Dutch Pathology Registry) for part of the data collection. The Hereditary Breast and Ovarian Cancer Research Group Netherlands (HEBON) consists of the following collaborating centres: coordinating centre: Netherlands Cancer Institute, Amsterdam, the Netherlands: M. A. Rookus, F. B. L. Hogervorst, F. E. van Leeuwen, S. Verhoef, M. K. Schmidt, N. S. Russell, J. L. de Lange, R. Wijnands; Erasmus Medical Center, Rotterdam, the Netherlands: J. M. Collée, A. M. W. van den Ouweland, M. J. Hooning, C. Seynaeve, C. H. M. van Deurzen, I. M. Obdeijn; Leiden University Medical Center, the Netherlands: C. J. van Asperen, J. T. Wijnen, R. A. E. M.
Tollenaar, P. Devilee, T. C. T. E. F. van Cronenburg; Radboud University Nijmegen Medical Center, the Netherlands: C. M. Kets, A. R. Mensenkamp; University Medical Center Utrecht, the Netherlands: M. G. E. M. Ausems, R. B. van der Luijt, C. C. van der Pol; Amsterdam Medical Center, Amsterdam, the Netherlands: C. M. Aalfs, T. A. M. van Os; VU University Medical Center, Amsterdam, Amsterdam, the Netherlands: J. J. P. Gille, Q. Waisfisz, H. E. J. Meijers-Heijboer; University Hospital Maastricht, the Netherlands: E. B. Gómez-Garcia, M. J. Blok; University Medical Center Groningen, the Netherlands: J. C. Oosterwijk, A. H. van der Hout, M. J. Mourits, G. H. de Bock; The Netherlands Foundation for the detection of hereditary tumours, Leiden, the Netherlands: $\mathrm{H}$. F. Vasen; The Netherlands Comprehensive Cancer Organization (IKNL): S. Siesling, J. Verloop; The Dutch Pathology Registry (PALGA): L. I. H. Overbeek. The HEBON study is supported by the Dutch Cancer Society grants NKI1998-1854, NKI2004-3088, NKI2007-3756, the Netherlands Organization of Scientific Research grant NWO 91109024, the Pink Ribbon grants 110005 and 2014187.WO76, the BBMRI grant NWO 184.021.007/CP46 and the Transcan grant JTC 2012 Cancer 12-054. HEBON thanks the registration teams of IKNL and PALGA for part of the data collection. HRBCP is supported by The Hong Kong Hereditary Breast Cancer Family Registry and the Dr. Ellen Li Charitable Foundation, Hong Kong. We the thank Hong Kong Sanatorium and Hospital for their continual support. HUNBOCS: Hungarian Breast and Ovarian Cancer Study was supported by Hungarian research grants KTIA-OTKA CK-80745, OTKA K-112228 and the Norwegian EEA Financial Mechanism Hu0115/NA/2008-3/OP-9. We thank the Hungarian Breast and Ovarian Cancer Study Group members (Janos Papp, Tibor Vaszko, Aniko Bozsik, Timea Pocza, Judit Franko, Maria Balogh, Gabriella Domokos, Judit Ferenczi, Department of Molecular Genetics, National Institute of Oncology, Budapest, Hungary) and the clinicians and patients for their contributions to this study. HVH: We thank the Oncogenetics Group (VHIO), and the High Risk and Cancer Prevention Unit of the University Hospital Vall d'Hebron. ICO: Contract grant sponsor: Asociación Española Contra el Cáncer, Spanish Health Research Fund; Carlos III Health Institute; Catalan Health Institute and Autonomous Government of Catalonia. Contract grant numbers: ISCIIIRETIC RD06/0020/1051, RD12/0036/008, PI10/01422, PI10/00748, PI13/ 00285, PIE13/00022, 2009SGR290 and 2014SGR364. We thank the ICO Hereditary Cancer Program team led by Dr. Gabriel Capella. The IHCC was supported by Grant PBZ_KBN_122/P05/2004. The ILUH group was supported by the Icelandic Association "Walking for Breast Cancer Research" and by the Landspitali University Hospital Research Fund. IOVHBOCS is supported by Ministero della Salute and " $5 \times 1000$ " Istituto Oncologico Veneto grant. IPOBCS: This study was in part supported by Liga Portuguesa Contra o Cancro. We thank Drs. Ana Peixoto, Catarina Santos, Patrícia Rocha and Pedro Pinto for their skilful contribution to the study. KConFab is supported by a grant from the National Breast Cancer Foundation, and previously by the National Health and Medical Research Council (NHMRC), the Queensland Cancer Fund, the Cancer Councils of New South Wales, Victoria, Tasmania and South Australia, and the Cancer Foundation of Western Australia; We thank Heather Thorne, Eveline Niedermayr, all the kConFab research nurses and staff, the heads and staff of the Family Cancer Clinics, and the Clinical Follow Up Study [which has received funding from the NHMRC, the National Breast Cancer Foundation, Cancer Australia, and the National Institutes of Health (USA)] for their contributions to this resource, and the many families who contribute to KConFab. KOHBRA is supported by a grant from the National R\&D Program for Cancer Control, Ministry for Health, Welfare and Family Affairs, Republic of Korea (1020350). MAYO is supported by NIH grants CA116167, CA128978 and CA176785, an $\mathrm{NCl}$ Specialized Program of Research Excellence (SPORE) in Breast Cancer (CA116201), a U.S. Department of Defense Ovarian Cancer Idea award (W81XWH-10-1-0341), a grant from the Breast Cancer Research Foundation, a generous gift from the David F. and Margaret T. Grohne Family Foundation and the Ting Tsung and Wei Fong Chao Foundation. MODSQUAD was supported by MH CZ - DRO (MMCI, 00209805) and by the European Regional Development Fund and the State Budget of the Czech Republic (RECAMO, CZ.1.05 /2.1.00/ 03.0101) to LF, and by Charles University in Prague project UNCE204024 (to MZ). MODSQUAD acknowledges ModSQuaD members Csilla Szabo (National Human Genome Research Institute, National Institutes of Health, Bethesda, MD, USA) and Michal Zikan, Petr Pohlreich and Zdenek Kleibl (Oncogynecologic Center and Department of Biochemistry and Experimental Oncology, First Faculty of Medicine, Charles University, Prague, Czech Republic). MSKCC is supported by grants from the Breast Cancer Research Foundation, the Robert and Kate Niehaus Clinical Cancer Genetics Initiative, and the Andrew Sabin Research Fund. NCI: The research of MHG and PLM was supported by the Intramural Research Program 
of the National Cancer Institute, $\mathrm{NIH}$, and by support services contracts NO2-CP-11019-50 and N02-CP-65504 with Westat, Inc., Rockville, MD, USA. NNPIO: This work has been supported by the Russian Federation for Basic Research (grants 13-04-92613, 14-04-93959 and 15-04-01744). OCGN: We thank members and participants in the Ontario Cancer Genetics Network for their contributions to the study. OSUCCG is supported by The Ohio State University Comprehensive Cancer Center. Leigha Senter, Kevin Sweet, Caroline Craven and Michelle O'Conor were instrumental in accrual of study participants, ascertainment of medical records and database management. Samples were processed by the OSU Human Genetics Sample Bank. PBCS: This work was supported by the ITT (Istituto Toscano Tumori) grants 2011-2013. SEABASS: Ministry of Science, Technology and Innovation, Ministry of Higher Education (UM.C/HIR/MOHE/06) and Cancer Research Initiatives Foundation. We thank Yip Cheng Har, Nur Aishah Mohd Taib, Phuah Sze Yee, Norhashimah Hassan and all the research nurses, research assistants and doctors involved in the MyBrCa Study for assistance in patient recruitment, data collection and sample preparation. In addition, we thank Philip lau, Sng Jen-Hwei and Sharifah Nor Akmal for contributing samples from the Singapore Breast Cancer Study and the HUKM-HKL Study, respectively. The Malaysian Breast Cancer Genetic Study is funded by research grants from the Malaysian Ministry of Science, Technology and Innovation, Ministry of Higher Education (UM.C/HIR/MOHE/06) and charitable funding from Cancer Research Initiatives Foundation. SWE-BRCA collaborators are supported by the Swedish Cancer Society. Swedish scientists participating as SWE-BRCA collaborators are as follows: from Lund University and University Hospital: Åke Borg, Håkan Olsson, Helena Jernström, Karin Henriksson, Katja Harbst, Maria Soller, Ulf Kristoffersson; from Gothenburg Sahlgrenska University Hospital: Anna Öfverholm, Margareta Nordling, Per Karlsson, Zakaria Einbeigi; from Stockholm and Karolinska University Hospital: Anna von Wachenfeldt, Annelie Liljegren, Annika Lindblom, Brita Arver, Gisela Barbany Bustinza, Johanna Rantala; from Umeå University Hospital: Beatrice Melin, Christina Edwinsdotter Ardnor, Monica Emanuelsson; from Uppsala University: Hans Ehrencrona, Maritta Hellström Pigg, Richard Rosenquist; from Linköping University Hospital: Marie Stenmark-Askmalm, Sigrun Liedgren. The University of Chicago is supported by NCI Specialized Program of Research Excellence (SPORE) in Breast Cancer (CA125183), R01 CA142996, 1U01CA161032 and by the Ralph and Marion Falk Medical Research Trust, the Entertainment Industry Fund National Women's Cancer Research Alliance and the Breast Cancer research Foundation. $\mathrm{OIO}$ is an American Cancer Society clinical research professor. We thank Cecilia Zvocec, Qun Niu, physicians, genetic counsellors, research nurses and staff of the Cancer Risk Clinic for their contributions to this resource, and the many families who contribute to our program. UCSF Cancer Risk Program and Helen Diller Family Comprehensive Cancer Center. We thank the following genetic counsellors for participant recruitment: Beth Crawford, Kate Loranger, Julie Mak, Nicola Stewart, Robin Lee, Amie Blanco and Peggy Conrad. Thanks are also extended to Ms. Salina Chan for her data management. UKFOCR was supported by a project grant from CRUK to Paul Pharoah. We thank Paul Pharoah, Simon Gayther, Susan Ramus, Carole Pye, Patricia Harrington and Eva Wozniak for their contributions to the UKFOCR. University of Pennsylvania: National Institutes of Health (NIH) (grants R01-CA102776 and R01-CA083855); Breast Cancer Research Foundation; Susan G. Komen Foundation for the Cure, Basser Research Center for BRCA. UPITT/MWH: Frieda G. and Saul F. Shapira BRCA-Associated Cancer Research Program; Hackers for Hope Pittsburgh. VFCTG: Victorian Cancer Agency, Cancer Australia, National Breast Cancer Foundation Geoffrey Lindeman, Marion Harris, Martin Delatycki of the Victorian Familial Cancer Trials Group. We thank Sarah Sawyer and Rebecca Driessen for assembling this data and Ella Thompson for performing all DNA amplification. WCP: BYK is funded by the American Cancer Society Early Detection Professorship (SIOP-06-258-01-COUN) and National Center for Advancing Translational Sciences (NCATS) grant UL1TR000124. Funding for the iCOGS infrastructure came from: the European Community's Seventh Framework Programme under grant agreement number 223175 (HEALTH-F2-2009-223175) (COGS), Cancer Research UK (C1287/A10118, C1287/A 10710, C12292/A11174, C1281/ A12014, C5047/A8384, C5047/A15007, C5047/A10692, C8197/A16565), the National Institutes of Health (CA128978) and Post-Cancer GWAS initiative (1U19 CA148537, 1 U19 CA148065 and 1 U19 CA148112 - the GAME-ON Initiative), the U.S. Department of Defense (W81XWH-10-1-0341), the Canadian Institutes of Health Research $(\mathrm{CIHR})$ for the CIHR Team in Familial Risks of Breast Cancer, Komen Foundation for the Cure, the Breast Cancer Research Foundation and the Ovarian Cancer Research Fund.

The funders had no role in the design and conduct of the study; collection, management, analysis and interpretation of data; preparation, review or approval of the manuscript; or the decision to submit the manuscript for publication.

\section{Author details}

${ }^{1}$ Department of Molecular Medicine, Sapienza University of Rome, Viale Regina Elena, 324, 00161 Rome, Italy. ${ }^{2}$ Centre for Cancer Genetic Epidemiology, Department of Public Health and Primary Care, School of Clinical Medicine, University of Cambridge, Cambridge, UK. ${ }^{3}$ Laboratory Medicine Program, University Health Network, Toronto, ON, Canada. ${ }^{4}$ Department of Laboratory Medicine and Pathobiology, University of Toronto, Toronto, ON, Canada. ${ }^{5}$ Department of Population Sciences, Beckman Research Institute of City of Hope, Duarte, CA, USA. ${ }^{6}$ Peter MacCallum Cancer Institute, East Melbourne, Australia. 'Women's Cancer Program at the Samuel Oschin Comprehensive Cancer Institute, Cedars-Sinai Medical Center, Los Angeles, CA, USA. ${ }^{8}$ Familial Cancer Centre, Peter MacCallum Cancer Centre, Melbourne, Australia. ${ }^{9}$ Department of Oncology, The University of Melbourne, Melbourne, VIC, Australia. ${ }^{10}$ University of Pittsburgh School of Medicine, Pittsburgh, PA, USA. ${ }^{11}$ UPMC Cancer Center, Pittsburgh, PA, USA. ${ }^{12}$ Department of Medicine, Abramson Cancer Center, Perelman School of Medicine at The University of Pennsylvania, Philadelphia, PA, USA. ${ }^{13}$ Department of Epidemiology and Biostatistics, Abramson Cancer Center, Perelman School of Medicine, University of Pennsylvania, Philadelphia, PA, USA. ${ }^{14}$ Department of Preventive Medicine, Keck School of Medicine, University of Southern California Norris Comprehensive Cancer Center, Los Angeles, CA, USA. ${ }^{15}$ Department of Medicine and Genetics, University of California, San Francisco, San Francisco, CA, USA. ${ }^{16}$ Center for Clinical Cancer Genetics and Global Health, University of Chicago Medical Center, Chicago, IL, USA. ${ }^{17}$ Department of Clinical Genetics, Karolinska University Hospital, Stockholm, Sweden. ${ }^{18}$ Cancer Research Initiatives Foundation, Sime Darby Medical Centre, Subang Jaya, Malaysia. ${ }^{19}$ University Malaya Cancer Research Institute, Faculty of Medicine, University Malaya Medical Centre, University Malaya, Kuala Lumpur, Malaysia. ${ }^{20}$ Section of Genetic Oncology, Department of Laboratory Medicine, University of Pisa and University Hospital of Pisa, Pisa, Italy. ${ }^{21}$ Department of Clinical Genetics, Vejle Hospital, Vejle, Denmark. ${ }^{22}$ Section of Molecular Diagnostics, Department of Biochemistry, Aalborg University Hospital, Aalborg, Denmark. ${ }^{23}$ Department of Clinical Genetics, Odense University Hospital, Odense C, Denmark. ${ }^{24}$ Department of Clinical Genetics, Aarhus University Hospital, Aarhus N, Denmark. ${ }^{25}$ Department of Molecular Virology, Immunology and Medical Genetics, College of Medicine, The Ohio State University, Columbus, $\mathrm{OH}$, USA. ${ }^{26}$ Division of Human Genetics, Department of Internal Medicine, The Comprehensive Cancer Center, The Ohio State University, Columbus, $\mathrm{OH}$, USA. ${ }^{27}$ Lunenfeld-Tanenbaum Research Institute, Mount Sinai Hospital, Toronto, ON, Canada. ${ }^{28}$ Department of Molecular Genetics, University of Toronto, Toronto, ON, Canada. ${ }^{29}$ Center for Medical Genetics, North Shore University Health System, Evanston, IL, USA. ${ }^{30}$ N.N. Petrov Institute of Oncology, St. Petersburg, Russia. ${ }^{31}$ Clinical Genetics Branch, Division of Cancer Epidemiology and Genetics, National Cancer Institute, National Institutes of Health, Rockville, MD, USA. ${ }^{32}$ Department of Obstetrics and Gynecology, Comprehensive Cancer Center, Medical University of Vienna, Vienna, Austria. ${ }^{33}$ Department of Urology, Medical University of Vienna, Vienna, Austria. ${ }^{34}$ Department of Medicine, Memorial Sloan-Kettering Cancer Center, New York, NY, USA. ${ }^{35}$ Clinical Genetics Service, Department of Medicine, Memorial Sloan-Kettering Cancer Center, New York, NY, USA. ${ }^{36}$ Department of Cancer Epidemiology and Genetics, Masaryk Memorial Cancer Institute, Brno, Czech Republic. ${ }^{37}$ Masaryk Memorial Cancer Institute and Faculty of Medicine, Masaryk University, Brno, Czech Republic.

${ }^{38}$ Department of Laboratory Medicine and Pathology, Mayo Clinic, Rochester, MN, USA. ${ }^{39}$ Department of Health Sciences Research, Mayo Clinic, Rochester, MN, USA. ${ }^{40}$ Department of Oncology, Mayo Clinic, Rochester, MN, USA. ${ }^{41}$ Department of Hematology and Oncology, University of Kansas Medical Center, Kansas City, KS, USA. ${ }^{42}$ Department of Surgery, Daerim St. Mary's Hospital, Seoul, Korea. ${ }^{43}$ Department of Genetics, Portuguese Institute of Oncology, Porto, Portugal. ${ }^{44}$ Biomedical Sciences Institute (ICBAS), University of Porto, Porto, Portugal. ${ }^{45}$ Immunology and Molecular Oncology Unit, Veneto Institute of Oncology IOV - IRCCS (Scientific Institute of Hospitalization and Care), Padua, Italy. ${ }^{46}$ Department of Pathology, Landspitali University Hospital and Biomedical Centre (BMC), Faculty of Medicine, University of Iceland, Reykjavik, Iceland. ${ }^{47}$ Department of Oncology, Landspitali University Hospital and Faculty of Medicine, University of Iceland, Reykjavik, Iceland. ${ }^{48}$ Department of Genetics and Pathology, Pomeranian 
Medical University, Szczecin, Poland. ${ }^{49}$ Genetic Counseling Unit, Hereditary Cancer Program, Biomedical Research Institute of Girona (IDIBGI), Catalan Institute of Oncology, Girona, Spain. ${ }^{50}$ Breast Cancer and Systems Biology Unit, Bellvitge Biomedical Research Institute (IDIBELL), Catalan Institute of Oncology, Barcelona, Spain. ${ }^{51}$ Department of Medical Oncology, Vall d'Hebron University Hospital, Barcelona, Spain. ${ }^{52}$ Oncogenetics Group, Vall d'Hebron University Hospital, Vall d'Hebron Institute of Oncology (VHIO) and Universitat Autònoma de Barcelona, Barcelona, Spain. ${ }^{53}$ Department of Pathology, National Institute of Oncology, Budapest, Hungary. ${ }^{54}$ Department of Molecular Genetics, National Institute of Oncology, Budapest, Hungary.

${ }^{55}$ The Hong Kong Hereditary Breast Cancer Family Registry, Cancer Genetics Center, Hong Kong Sanatorium and Hospital, Hong Kong, China.

${ }^{56}$ Department of Surgery, The University of Hong Kong, Hong Kong, China.

${ }^{57}$ Department of Obstetrics and Gynecology, University of Helsinki and Helsinki University Hospital, Helsinki, Finland. ${ }^{58}$ Department of Clinical Genetics, University of Helsinki and Helsinki University Hospital, Helsinki, Finland. ${ }^{59}$ Department of Oncology, San Carlos Clinical Hospital Health Research Institute (IdISSC), San Carlos Clinical Hospital, Madrid, Spain. ${ }^{60}$ Molecular Oncology Laboratory, San Carlos Clinical Hospital Health Research Institute (IdISSC), San Carlos Clinical Hospital, Madrid, Spain. ${ }^{61}$ Center for Medical Genetics, Ghent University, Ghent, Belgium. ${ }^{62}$ Lombardi Comprehensive Cancer Center, Georgetown University, Washington, DC, USA. ${ }^{63}$ Department of Tumour Biology, Institut Curie, Paris, France. ${ }^{64}$ CNRS UMR5558, Université Lyon 1, Lyon, France. ${ }^{65}$ Unité de Prévention et d'Epidémiologie Génétique, Centre Léon Bérard, Lyon, France. ${ }^{66}$ Université Paris Descartes, Sorbonne Paris Cité, Paris, France. ${ }^{67}$ INSERM U1052, CNRS UMR5286, Centre de Recherche en Cancérologie de Lyon, Université Lyon, Lyon, France. ${ }^{68}$ Department of Gynaecology and Obstetrics, Technical University of Munich, Munich, Germany. ${ }^{69}$ Institute of Human Genetics, University of Wurzburg, Wurzburg, Germany. ${ }^{70}$ Institute of Human Genetics, University Hospital Heidelberg, Heidelberg, Germany. ${ }^{71}$ Institute for Medical Informatics, Statistics and Epidemiology University of Leipzig, Leipzig, Germany. ${ }^{72}$ University of Dusseldorf, Dusseldorf, Germany. ${ }^{73}$ Hannover Medical School, Hannover, Germany. ${ }^{74}$ Center for Hereditary Breast and Ovarian Cancer, Center for Integrated Oncology (ClO) and Center for Molecular Medicine Cologne (CMMC), Medical Faculty, University of Cologne and University Hospital Cologne, Cologne, Germany. ${ }^{75}$ Department of Gynecology and Obstetrics, Technical University of Dresden, Dresden, Germany. ${ }^{76}$ Department of Gynaecolgy and Obstetrics, University Hospital of Schleswig-Holstein, Christian-Albrechts-University of Kiel, Kiel, Germany. ${ }^{77}$ Institute of Human Genetics, Charité, Berlin, Germany. ${ }^{78}$ Institute of Human Genetics, Leipzig, Germany. ${ }^{79}$ Department of Pathology and Laboratory Medicine, University of Kansas Medical Center, Kansas City, KS, USA. ${ }^{80}$ Genetic Medicine, Manchester Academic Health Sciences Centre, Central Manchester University Hospitals NHS Foundation Trust, Manchester, UK. ${ }^{81}$ Yorkshire Regional Genetics Service, Leeds, UK. ${ }^{82}$ Clinical Genetics, Guy's and St. Thomas' NHS Foundation Trust, London, UK. ${ }^{83}$ Oncogenetics Team, The Institute of Cancer Research and Royal Marsden NHS Foundation Trust, Sutton, UK. ${ }^{84}$ Molecular Genetics of Breast Cancer, German Cancer Research Center (DKFZ), Heidelberg, Germany. ${ }^{85}$ Cancer Risk and Prevention Clinic, Dana-Farber Cancer Institute, Boston, MA, USA. ${ }^{86}$ Molecular Diagnostics Laboratory, Institute of Nuclear and Radiological Sciences and Technology (INRASTES), National Centre for Scientific Research "Demokritos", Aghia Paraskevi Attikis, Athens, Greece. ${ }^{87}$ Department of Medical Oncology, Papageorgiou Hospital, Aristotle University of Thessaloniki School of Medicine, Thessaloniki, Greece. ${ }^{88}$ Department of Medical Science, University of Turin, Turin, Italy. ${ }^{89} \mathrm{AO}$ Città della Salute e della Scienza, Turin, Italy. ${ }^{90}$ Section of Medical Oncology, Department of Surgical and Oncological Sciences, University of Palermo, Palermo, Italy. ${ }^{91}$ Department of Oncology and Haematology, University of Modena and Reggio Emilia, Modena, Italy. ${ }^{92}$ Unit of Medical Genetics, Department of Biomedical, Experimental and Clinical Sciences, University of Florence, Florence, Italy. ${ }^{93}$ Unit of Hereditary Cancer, Department of Epidemiology, Prevention and Special Functions, IRCCS (Scientific Institute of Hospitalization and Care), AOU San Martino - IST National Institute for Cancer Research, Genoa, Italy. ${ }^{94}$ Molecular and Nutritional Epidemiology Unit, Cancer Research and Prevention Institute (ISPO), Florence, Italy. ${ }^{95}$ Unit of Genetic Counselling, Medical Oncology Department, Regina Elena National Cancer Institute, Rome, Italy. ${ }^{96}$ Unit of Molecular Bases of Genetic Risk and Genetic Testing, Department of Preventive and Predictive Medicine, IRCCS (Scientific Institute of Hospitalization and Care), National Cancer Institute (INT), 20133 Milan, Italy.
${ }^{97}$ Unit of Medical Genetics, Department of Preventive and Predictive Medicine, IRCCS (Scientific Institute of Hospitalization and Care), National Cancer Institute (INT), Milan, Italy. ${ }^{98}$ Division of Cancer Prevention and Genetics, European Institute of Oncology (IEO), Milan, Italy. ${ }^{99}$ Division of Experimental Oncology, CRO Aviano National Cancer Institute, Aviano, PN, Italy. ${ }^{100}$ IFOM, FIRC (Italian Foundation for Cancer Research) Institute of Molecular Oncology, Milan, Italy. ${ }^{101}$ Cogentech Cancer Genetic Test Laboratory, Milan, Italy. ${ }^{102}$ National Cancer Institute "Giovanni Paolo II", Bari, Italy. ${ }^{103}$ Clinical Cancer Genetics, City of Hope Clinical Cancer Genetics Community Research Network, Duarte, CA, USA. ${ }^{104}$ Human Genetics Group, Human Cancer Genetics Program, Spanish National Cancer Centre (CNIO), Madrid, Spain. ${ }^{105}$ Biomedical Network on Rare Diseases (CIBERER), Madrid, Spain. ${ }^{106}$ Human Genetics Group, Spanish National Cancer Centre (CNIO), Madrid, Spain. ${ }^{107}$ Human Genotyping (CEGEN) Unit, Human Cancer Genetics Program, Spanish National Cancer Research Centre (CNIO), Madrid, Spain. ${ }^{108}$ Cancer Division, QIMR Berghofer Medical Research Institute, Brisbane, Australia. ${ }^{109}$ Department of Clinical Genetics, Rigshospitalet, Copenhagen University Hospital, Copenhagen, Denmark. ${ }^{110}$ Department of Oncology, Rigshospitalet, Copenhagen University Hospital, Copenhagen, Denmark. ${ }^{111}$ Center for Genomic Medicine, Rigshospitalet, Copenhagen University Hospital, Copenhagen, Denmark. ${ }^{112}$ Department of Medical Oncology, Beth Israel Deaconess Medical Center, Boston, MA, USA. ${ }^{113}$ State Research Institute Centre for Innovative Medicine, Vilnius, Lithuania. ${ }^{114}$ Department of Dermatology, Huntsman Cancer Institute, University of Utah School of Medicine, Salt Lake City, UT, USA. ${ }^{115}$ Department of Medicine, Huntsman Cancer Institute, University of Utah School of Medicine, Salt Lake City, UT, USA. ${ }^{116}$ Department of Clinical Genetics, Fox Chase Cancer Center, Philadelphia, PA, USA. ${ }^{117}$ Department of Pathology \& Molecular Medicine, Juravinski Hospital and Cancer Centre, McMaster University, Hamilton, ON, Canada. ${ }^{118}$ Department of Epidemiology, Mailman School of Public Health, Columbia University, New York, NY, USA. ${ }^{119}$ Department of Epidemiology, Cancer Prevention Institute of California, Fremont, CA, USA. ${ }^{120}$ Genetic Epidemiology Laboratory, Department of Pathology, University of Melbourne, Parkville, Australia.

Received: 28 September 2015 Accepted: 6 January 2016 Published online: 09 February 2016

\section{References}

1. Ly D, Forman D, Ferlay J, Brinton LA, Cook MB. An international comparison of male and female breast cancer incidence rates. Int J Cancer. 2013;132(8):1918-26.

2. Korde LA, Zujewski JA, Kamin L, Giordano S, Domchek S, Anderson WF, et al. Multidisciplinary meeting on male breast cancer: summary and research recommendations. J Clin Oncol. 2010;28(12):2114-22.

3. Basham VM, Lipscombe JM, Ward JM, Gayther SA, Ponder BA, Easton DF, et al. BRCA1 and BRCA2 mutations in a population-based study of male breast cancer. Breast Cancer Res. 2002;4:R2.

4. Ottini L, Masala G, D'Amico C, Mancini B, Saieva C, Aceto G, et al. BRCA1 and BRCA2 mutation status and tumor characteristics in male breast cancer: a population-based study in Italy. Cancer Res. 2003;63(2):342-7.

5. Rizzolo P, Silvestri V, Tommasi S, Pinto R, Danza K, Falchetti M, et al. Male breast cancer: genetics, epigenetics, and ethical aspects. Ann Oncol. 2013;24 Suppl 8:viii75-82.

6. Breast Cancer Linkage Consortium. Cancer risks in BRCA2 mutation carriers. J Natl Cancer Inst. 1999;91(15):1310-6.

7. Thompson D, Easton DF, Breast Cancer Linkage Consortium. Cancer incidence in BRCA1 mutation carriers. J Natl Cancer Inst. 2002;94(18):1358-65.

8. Tai YC, Domchek S, Parmigiani G, Chen S. Breast cancer risk among male BRCA1 and BRCA2 mutation carriers. J Natl Cancer Inst. 2007;99(23):1811-4.

9. Evans DG, Susnerwala I, Dawson J, Woodward E, Maher ER, Lalloo F. Risk of breast cancer in male BRCA2 carriers. J Med Genet. 2010;47(10):710-1.

10. Brinton LA, Key TJ, Kolonel LN, Michels KB, Sesso HD, Ursin G, et al. Prediagnostic sex steroid hormones in relation to male breast cancer risk. J Clin Oncol. 2015;33(18):2041-50.

11. Anderson WF, Jatoi I, Tse J, Rosenberg PS. Male breast cancer: a populationbased comparison with female breast cancer. J Clin Oncol. 2010;28:232-9.

12. Johansson I, Killander F, Linderholm B, Hedenfalk I. Molecular profiling of male breast cancer - lost in translation? Int J Biochem Cell Biol. 2014;53:526-35. 
13. Mavaddat N, Barrowdale D, Andrulis IL, Domchek SM, Eccles D, Nevanlinna $\mathrm{H}$, et al. Pathology of breast and ovarian cancers among BRCA1 and BRCA2 mutation carriers: results from the Consortium of Investigators of Modifiers of BRCA1/2 (CIMBA). Cancer Epidemiol Biomarkers Prev. 2012;21(1):134-47.

14. Kwiatkowska E, Teresiak M, Filas V, Karczewska A, Breborowicz D, Mackiewicz A. BRCA2 mutations and androgen receptor expression as independent predictors of outcome of male breast cancer patients. Clin Cancer Res. 2003;9(12):4452-9.

15. Ding YC, Steele L, Kuan CJ, Greilac S, Neuhausen SL. Mutations in BRCA2 and PALB2 in male breast cancer cases from the United States. Breast Cancer Res Treat. 2011;126(3):771-8.

16. Ottini L, Silvestri V, Rizzolo P, Falchetti M, Zanna I, Saieva C, et al. Clinical and pathologic characteristics of BRCA-positive and BRCA-negative male breast cancer patients: results from a collaborative multicenter study in Italy. Breast Cancer Res Treat. 2012:134(1):411-8.

17. Deb S, Jene N, kConFab Investigators, Fox SB. Genotypic and phenotypic analysis of familial male breast cancer shows under representation of the HER2 and basal subtypes in BRCA-associated carcinomas. BMC Cancer. 2012:12:510

18. Chenevix-Trench G, Milne RL, Antoniou AC, Couch FJ, Easton DF, Goldgar $D E$, et al. An international initiative to identify genetic modifiers of cancer risk in BRCA1 and BRCA2 mutation carriers: the Consortium of Investigators of Modifiers of BRCA1 and BRCA2 (CIMBA). Breast Cancer Res. 2007;9:104.

19. Edge S, Byrd DR, Compton CC, Fritz AG, Greene FL, Trotti A, editors. AJCC cancer staging manual. 6th ed. New York: Springer; 2002.

20. U.S. Department of Health and Human Services, National Cancer Institute, Surveillance, Epidemiology, and End Results (SEER) Program. Incidence SEER 18 Registries Research Data + Hurricane Katrina Impacted Louisiana Cases, November 2013 Submission (1973-2011 varying) - Linked to County Attributes - Total U.S., 1969-2012 Counties. Bethesda, MD: National Cancer Institute, Division of Cancer Control and Prevention Sciences, Surveillance Research Program, Surveillance Systems Branch; released April 2014 [updated 7 May 2014]. http://seer.cancer.gov/data/seerstat/nov2013/. Accessed 22 January 2016.

21. Friedman LS, Gayther SA, Kurosaki T, Gordon D, Noble B, Casey G. Mutation analysis of BRCA1 and BRCA2 in a male breast cancer population. Am J Hum Genet. 1997;60(2):313-9.

22. Ottini L, Rizzolo P, Zanna I, Falchetti M, Masala G, Ceccarelli K, et al. BRCA1/ BRCA2 mutation status and clinical-pathologic features of 108 male breast cancer cases from Tuscany: a population-based study in central Italy. Breast Cancer Res Treat. 2009;116(3):577-86.

23. Giordano SH, Cohen DS, Buzdar AU, Perkins G, Hortobagyi GN. Breast carcinoma in men: a population-based study. Cancer. 2004;101(1):51-7.

24. Anders CK, Fan C, Parker JS, Carey LA, Blackwell KL, Klauber-DeMore N, et al. Breast carcinomas arising at a young age: unique biology or a surrogate for aggressive intrinsic subtypes? J Clin Oncol. 2011;29(1):e18-20.

25. Masci G, Caruso M, Caruso F, Salvini P, Carnaghi C, Giordano L, et al. Clinicopathological and immunohistochemical characteristics in male breast cancer: a retrospective case series. Oncologist. 2015;20(6):586-92.

26. Moten A, Obirieze A, Wilson LL. Characterizing lobular carcinoma of the male breast using the SEER database. J Surg Res. 2013;185(2):e71-6.

27. Foulkes WD, Metcalfe K, Sun P, Hanna WM, Lynch HT, Ghadirian P, et al. Estrogen receptor status in BRCA1- and BRCA2-related breast cancer: the influence of age, grade, and histological type. Clin Cancer Res. 2004;10(6):2029-34.

28. Kaplan JS, Schnitt SJ, Collins LC, Wang Y, Garber JE, Montgomery K, et al, Pathologic features and immunophenotype of estrogen receptor-positive breast cancers in BRCA1 mutation carriers. Am J Surg Pathol. 2012;36(10):1483-8.

29. Lim E, Vaillant F, Wu D, Forrest NC, Pal B, Hart AH, et al. Aberrant luminal progenitors as the candidate target population for basal tumor development in BRCA1 mutation carriers. Nat Med. 2009;15(8):907-13.

30. Rakha EA, Reis-Filho JS, Baehner F, Dabbs DJ, Decker T, Eusebi V, et al. Breast cancer prognostic classification in the molecular era: the role of histological grade. Breast Cancer Res. 2010;12:207.

\section{Submit your next manuscript to BioMed Central and we will help you at every step:}

- We accept pre-submission inquiries

- Our selector tool helps you to find the most relevant journal

- We provide round the clock customer support

- Convenient online submission

- Thorough peer review

- Inclusion in PubMed and all major indexing services

- Maximum visibility for your research

Submit your manuscript at www.biomedcentral.com/submit
() BioMed Central 